\title{
Analysis of interference in a double interchange heterozygote of wheat (Triticum aestivum L.)
}

\author{
Araceli Fominaya \\ and Nicolas Jouve*
}

\begin{abstract}
Department of Genetics, Faculty of Sciences, University of Alcalá de Henares Apdo. 20, Alcalá de Henares, Madrid, Spain.
\end{abstract}

A cytological analysis of meiotic configurations in a double interchange heterozygote in common wheat, Triticum aestivum L., involving 3DL/4DS and 1AL/UN (unidentified) chromosomes is presented, where pollen mother cells were stained with Giemsa. The translocations were identified by $\mathrm{C}$-banding and this staining procedure located the positions of chiasmata in meiotic configurations in both translocation complexes. A strong interference was found between the presence of chiasmata in the interstitial and neighbouring translocated segments. If a chiasma is present in the former, it excludes the simultaneous presence of one in the latter and vice versa. It was also observed that the simultaneous presence of chiasmata in both distal segments of a translocation quadrivalent is more frequent than other situations.

\section{INTRODUCTION}

In structural heterozygotes for a reciprocal translocation between two chromosome pairs, one pair of normal and one pair of heterozygous interchange arms occur. Both interchange arms consist of two segments separated by the break point. Thus a total of six regions can be considered for a quantitative analysis of the pairing and recombination potentials of the chromosomes involved in quadrivalent associations. However, this analysis is only meaningful when pairing can be individually studied in all six segments of the two sets present in the quadrivalent associations.

Usually, the cytomorphological differentiation between the chromosomes is insufficient to satisfy the recognition requirements of the abovementioned regions. Some models to estimate the frequencies of expected configurations from meiotic observations, avoiding the direct distinction of those regions, have been proposed by Sybenga $(1966 ; 1970 ; 1975)$. In recent years, Giemsa staining techniques have been developed and applied which enable the meiotic chromosomes of the B-genome, together with chromosomes $4 \mathrm{~A}$ and $7 \mathrm{~A}$, to be readily distinguished from others in common wheat, Triticum aestivum $\mathrm{L}$. (Gill and Kimber, 1974; Gerlach, 1977; Jewell, 1979; Seal, 1982; Armstrong, 1982; Van Niekerk

\footnotetext{
* To whom all correspondence should be sent.
}

and Pienaar, 1983; Ferrer et al., 1984a and $b$; Jouve et al., 1985).

This paper describes the application of the C-banding technique to map the different regions of the chromosomes involved in two reciprocal interchanges in common wheat. The mechanism of interference across the break point is analysed on the basis of comparisons of directly observed frequencies of the different multivalent associations with those expected if random placement of chiasmata occurs in the various segments of the multivalents.

\section{MATERIALS AND METHODS}

Meiotic observations at first metaphase of at least 600 pollen mother cells (PMCs) were made in plants of the intervarietal hybrid between the common wheat "Chinese Spring" and the Spanish cultivar "Alonso Peña 114" ("AP-114"). Using the monosomic series of "Chinese Spring" two important translocations involving the $3 \mathrm{D}, 4 \mathrm{D}, 1 \mathrm{~A}$ and another unidentified (UN) chromosome have been detected in this cross.

All the $F_{1}$ plants were double interchange heterozygotes and formed a maximum number of two quadrivalents (3D/4D and $1 \mathrm{~A} / \mathrm{UN})$ per PMC.

Anthers for meiotic analysis were collected from hybrid plants and were fixed in acetic 
acid-alcohol 1:3. Squash preparations of the fixed material were stained following a Giemsa Cbanding technique previously reported (Jouve et al., 1980).

\section{RESULTS AND DISCUSSION}

\section{C-banding characterization of chromosomes involved in the translocations.}

Two translocations which differed in the frequencies and type of chromosome associations were found in the hybrid. The chromosomes involved in the interchanges were previously identified using monosomics of "Chinese Spring" as cytological markers. Only "Chinese Spring" monosomics 3D, 4D and $1 \mathrm{~A} \times$ " $\mathrm{AP}-114$ " showed meiotic configurations of 19 bivalents +1 trivalent, (without univalents) or 17 bivalents +1 trivalent + 1 quadrivalent (without univalents). The main translocation was assigned to the chromosomes 3D and 4D which showed similar frequencies for the same critical meiotic configurations in the monosomics hybrids. Only one pair of the chromosomes involved in the second interchange was identified (1A); the other pair will be referred to as UN (unidentified) in the text.

By using the Giemsa C-banding technique it was possible to characterise the chromosome arms involved in each translocation. A good correspondence in the pattern of C-bands for the 3D, 4D and $1 \mathrm{~A}$ chromosomes between meiotic cells identified here and somatic idiograms previously described in "Chinese Spring" (Gill and Kimber, 1974; Iordansky et al., 1978; Van Niekerk and Pienaar, 1983; Endo and Gill, 1984) was found.

3DL/4DS-A total number of 672 PMCs were

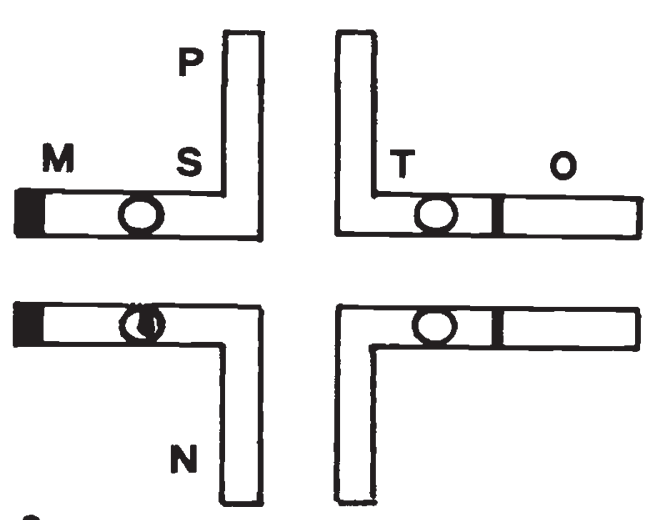

scored. The interchange involves to long arm of chromosome 3D and the short arm of chromosome $4 D .3 D L=34.96$ per cent and $4 \mathrm{DS}=19.86$ per cent of their relative chromosome length according the data of Sears, 1954, and Sallee and Kimber, 1979. Segments between centromeres in the ring-of-four chromosomes were almost identical in length. The interchange formed a ring quadrivalent in about 48 per cent of the cells and a chain quadrivalent in 14 per cent. The $3 \mathrm{D}$ chromosome is submetacentric and exhibits one telomeric band in its short arm (non-translocated). Chromosome 4D is also submetacentric and presents a faint intercalary band near the centromere in the proximal half of its long arm (non-translocated) (fig. 1a).

$1 \mathrm{AL} / \mathrm{UN}-\mathrm{A}$ total number of 600 PMCs were scored. This translocation was observed as a chainquadrivalent in about 23 per cent of the cells. The reciprocal interchange involved the long arm of the $1 \mathrm{~A}$ chromosome $(1 \mathrm{AL}=39.11$ per cent of the chromosome length). The chromosome $1 \mathrm{~A}$ is submetacentric and exhibits differential heterochromatic regions in the hybrids. One of the chromosomes showed a characteristic thin band in the proximal half of the short arm. The unidentified chromosome (UN) showed one faint terminal band in its non-translocated arm (fig. 1b).

\section{Meiotic configurations at first metaphase (MI)}

The C-banding staining procedure made it possible to distinguish the different regions of the chromosomes involved in quadrivalents, trivalents, bivalents and univalents (fig. $1 \mathrm{a}$ and $\mathrm{b}$ and fig. 2). Four different segments ( $M$ and $O$ non-translocated and $S$ and $T$ interstitial regions) can be recognised and their pairing frequencies
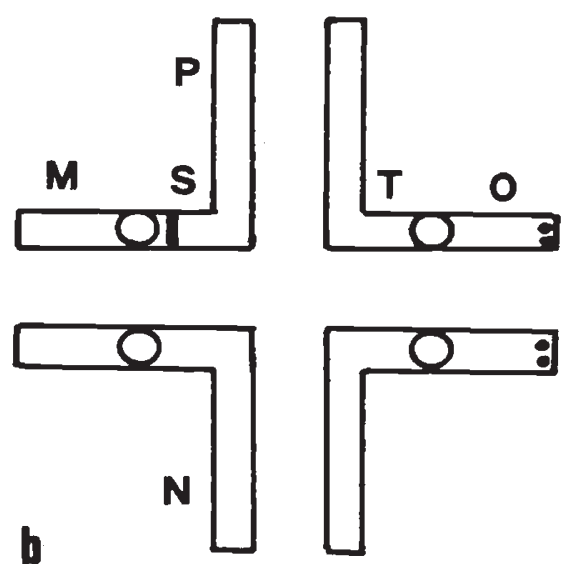

Figure 1 Diagramatic representation of the configuration types of chromosome associations for the 3DL/4DS (a) and 1AL/UN (b) reciprocal translocations illustrating distribution of C-bands and total number of PMCs per configuration. 


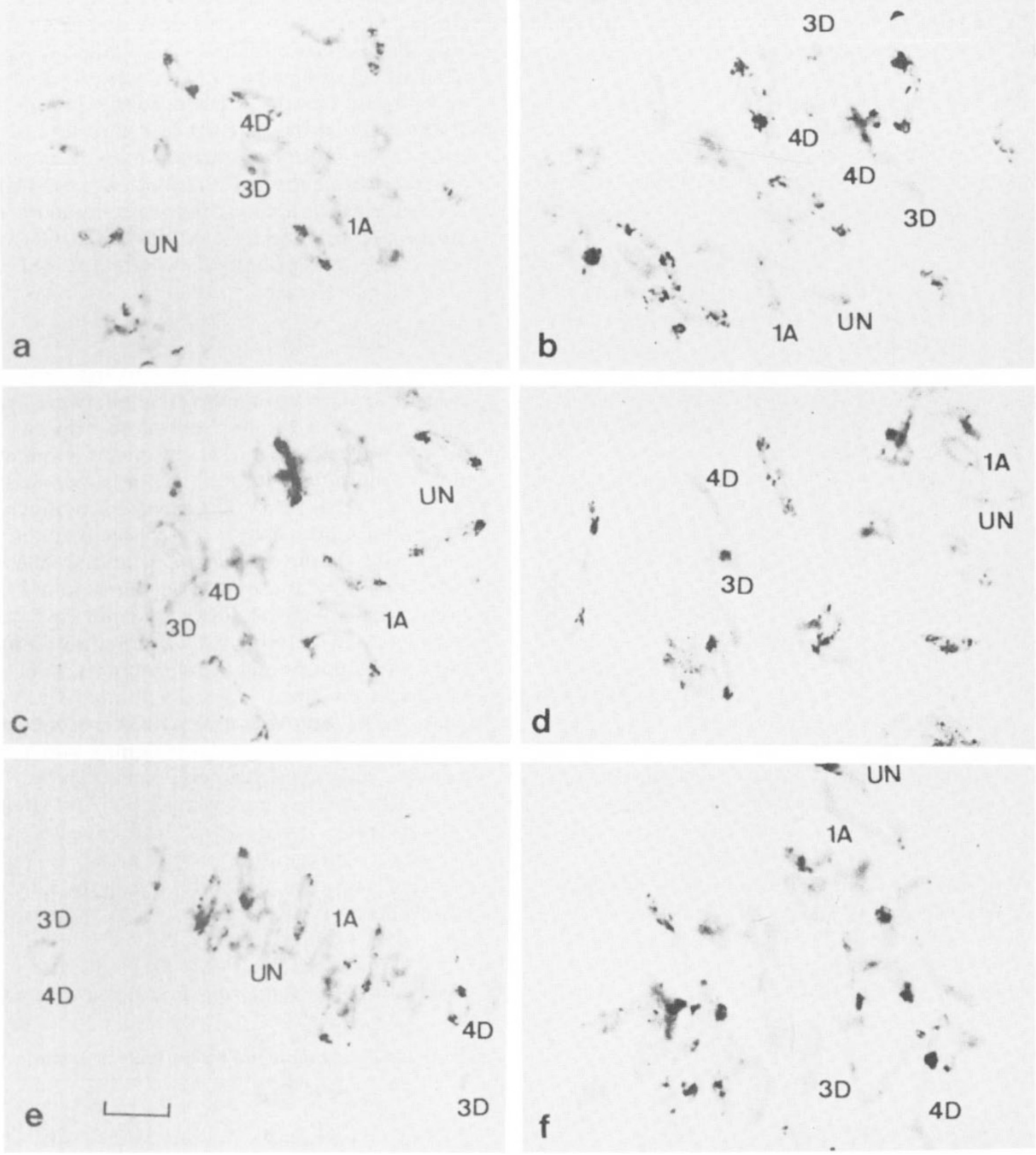

Figure 2 Photomicrographs of PMCs at first metaphase stained by Giemsa in a double reciprocal translocation heterozygote involving $3 \mathrm{DL} / 4 \mathrm{DS}$ and $1 \mathrm{AL} / \mathrm{UN}$ common wheat chromosomes. Bar represents $10 \mu \mathrm{m}$.

$3 D$ and $4 D$ appear as a ring quadrivalent (a); a chain quadrivalent unpaired at the $O(\mathrm{~d}), N$ or $P(\mathrm{f})$ or $M(\mathrm{c})$ non translocated regions; two open bivalents combining non homologous centromeres (e); or as four univalents (b).

$1 A$ and $U N$ appear as a chain quadrivalent unpaired at the 0 non translocated region (b); or as one trivalent bound by the $S$ region (f); or as two ring bivalents bound by their interstitial and non translocated regions (c to e); or as one $1 \mathrm{~A}$ open bivalent bound by its interstitial regions and one UN ring bivalent bound by non translocated and interstitial segments (a). 
scored for the 3DL/4DS interchange. All the six different segments ( $M$ and $O$ non-translocated, $S$ and $T$ interstitial, and $P$ and $N$ translocated regions) can be recognised for the $1 \mathrm{AL} / \mathrm{UN}$ interchange.

The different configurations that appeared at metaphase I are dependent on the location of the chiasmata. Thus, the positions of chiasmata were mapped and direct estimation of the pairing frequencies of each region was carried out (tables 1 and 2).

In each segment, chiasmata may either be present or absent. Thus, there are $2^{6}$ or 64 combinations. Each combination corresponds with one metaphase I configuration. According with Sybenga (1975), if the different chromosomes in the configurations cannot be recognised a number of these configurations have the same general shape. In the case of metacentric chromosomes the maximum number of different chromosome associations which can be distinguished is $16 ; 10$ as multivalents, and six combining bivalents and/or univalents. C-banding permits the differentiation of many configurations having the same external shape. This was particulary useful in the present study for the following configurations:

$-3 \mathrm{DL} / 4 \mathrm{DS}$ three types in the class of chain-offour chromosomes, and six types in the class of bivalents and/or univalents.

$-1 \mathrm{AL} / \mathrm{UN}$ two types in the class of chainof-four, two types in the class of chain-ofthree + univalent, seven types in the class of two bivalents and four types in the class of one bivalent + two univalents.

No multivalent configurations with chiasmata in the interstitial regions ( $S$ and $T$ ) were observed. However, they were very common in the bivalents. A similar situation was recently reported in lentil, Lens culinaris, by Buruchin and Ladizinsky (1983).
The expected frequency of each different configuration can be estimated as the product of the probabilities of each segment which is paired or unpaired, depending on what applies for each particular configuration. The observed and expected values for the different configurations which were classified by their general shape are given in table 3. The significant deviations existing between observed and expected distributions could be considered as an indication of interference in the translocation complexes. $\left(\chi^{2}=745.71 ; P<0.001\right.$ for 3DL/4DS; $\chi^{2}=670 \cdot 52 ; P<0 \cdot 001$, for $\left.1 \mathrm{AL} / \mathrm{UN}\right)$.

\section{Interference}

For reasons of simplicity and to state more precisely the existence of interference between specific segments, a grouping of configurations into categories in which only the four end segments are involved $(M, O, P$ and $N)$ was proposed by Sybenga $(1970,1975)$. The espected frequencies of all configurations are expressed as a function of the pairing by the end segments and are classified as follows: I. with four end segments paired (types 1 and 2 of table 3 ); II. with three of the four end segments paired (types 3, 4 and 5 of table 3); III. with two adjacent end segments paired, the other two unpaired (types 7, 8 and 9 of table 3 ); IV. with two opposite end segments paired (types 6 and all configurations having two bivalents); and V. those with one or no end segments paired (type 10 and configurations having 1 bivalent +2 univalents or 4 univalents). The observed and expected values for each configuration type grouped by this criterion are given in table 4. Highly significant deviations were observed for both translocations (for 3DL/4DS: $\chi^{2}=395.9, \quad P<0.001 ; \quad$ for 1AL/UN: $\left.\chi^{2}=235 \cdot 5, P<0 \cdot 001\right)$.

The effect of reduction of pairing in proximal

Table 1 The number of cells with chiasmata in the six segments of the 3DL/4DS translocations heterozygote of common wheat

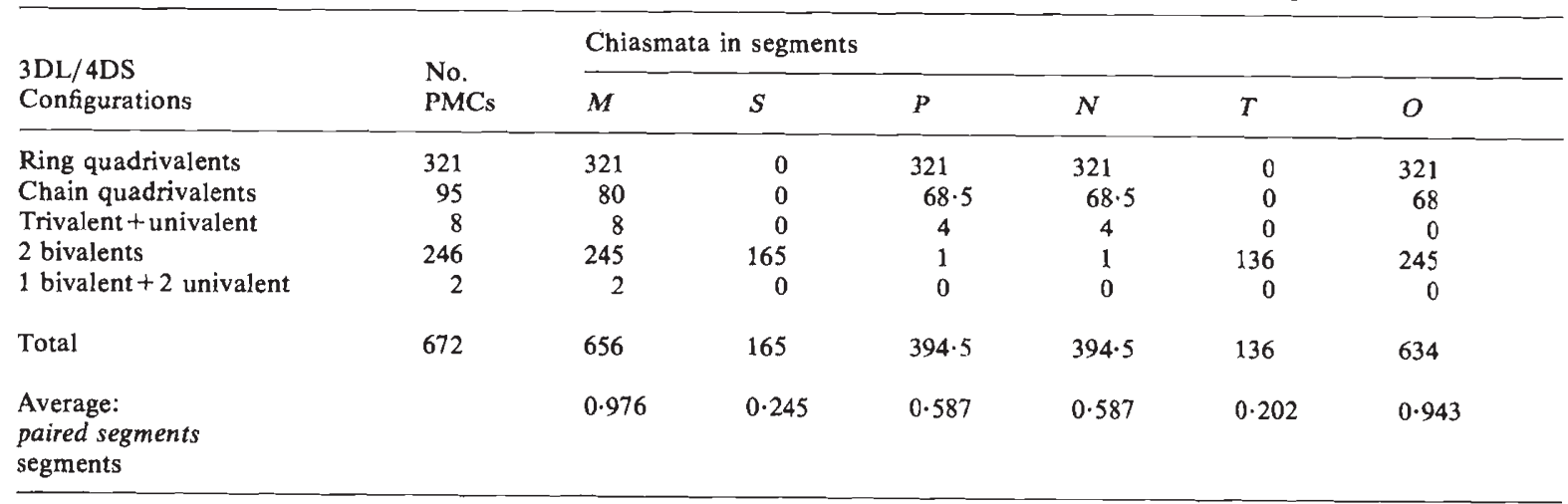


Table 2 The number of cells with chiasmata in the six segments of the 1AL/UN translocation heterozygote of common wheat

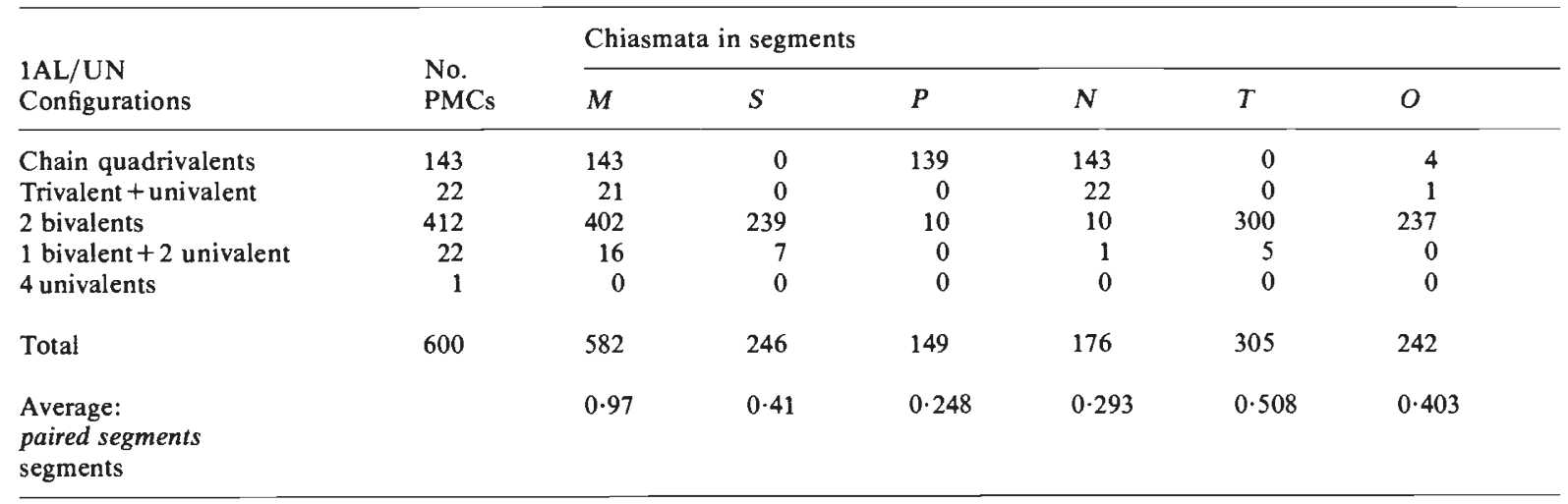

regions (interstitial) in multivalents with respect to bivalents observed in the material analysed here is similar to the reduction of the amount of crossing-over near the centromere in telocentric chromosomes of wheat observed by Sears (1972). Endrizzi and Kohel (1966) have shown a remarkably similar situation in cotton. In an analysis of the pairing of wheat telocentric chromosomes made by Sallee and Kimber (1979) the existence was demonstrated of some selection pressure for a minimum number of chiasmata per arm or per chromosome irrespective of its length. The pairing frequencies can be reduced drastically as a consequence of an overlapping effect of both factors, the separation into two different segments of a translocated arm and the interference between them across the break point in multivalents. The chiasmata to be formed could be shifted to terminal

Table 3 The observed frequencies of the configurations in the PMCs of the heterozygotes of the reciprocal translocations $3 \mathrm{DL} / 4 \mathrm{DS}$ and $1 \mathrm{AL} / \mathrm{UN}$ and the expected frequencies when the probabilities of pairing by $M, S, P, N, T$ and $O$ correspond with those given in tables 1 and 2

\begin{tabular}{|c|c|c|c|c|}
\hline \multirow[b]{2}{*}{ Configuration } & \multicolumn{2}{|c|}{$3 \mathrm{DL} / 4 \mathrm{DS}$} & \multicolumn{2}{|c|}{$1 \mathrm{AL} / \mathrm{UN}$} \\
\hline & Obs. & Exp. & Obs. & Exp. \\
\hline$\hat{v} \hat{v}$ & 0 & $95 \cdot 9$ & 0 & $32 \cdot 1$ \\
\hline$[7$ & 321 & 125 & 0 & $4 \cdot 3$ \\
\hline$\hat{\hat{n}_{v}}$ & 95 & $193 \cdot 1$ & 143 & $30 \cdot 4$ \\
\hline$\hat{v}^{n} v$ & 0 & $101 \cdot 3$ & 0 & $53 \cdot 2$ \\
\hline$\hat{v}$ & 0 & $10 \cdot 5$ & 0 & $49 \cdot 4$ \\
\hline$x$ & 0 & $0 \cdot 2$ & 0 & $4 \cdot 2$ \\
\hline$u^{\wedge}$ & 0 & $2 \cdot 5$ & 0 & 1.6 \\
\hline $8 \hat{v}$ & 0 & $5 \cdot 9$ & 0 & $26 \cdot 1$ \\
\hline $9 \wedge \wedge_{\lrcorner}$ & 8 & $19 \cdot 3$ & 22 & $36 \cdot 4$ \\
\hline 10 ฯ & 0 & $5 \cdot 1$ & 0 & $38 \cdot 6$ \\
\hline $\begin{array}{l}\text { 11-16 Biv. or } \\
\text { Biv. + univ. }\end{array}$ & 248 & $113 \cdot 2$ & 435 & $323 \cdot 7$ \\
\hline Total & 672 & 672 & 600 & 600 \\
\hline
\end{tabular}

(translocated) regions in the multivalents, whereas they could be formed in proximal segments (interstitial) in the bivalents.

A new classification of the configuration types observed in our material based on pairing behaviour of both interstitial and translocated regions instead of end segments can be more illustrative and is given in table 5 . The data presented in this table permit a better understanding of the interference mechanism in the heterozygotes. It is clear that the observed frequency of configurations having simultaneously chiasmata in the translocated and interstitial segments is lower than expected. This demonstrates the existence of a strong interference between the presence of chiasmata in the interstitial and neighbouring translocated segments. If a chiasma is present in the former, it excludes the simultaneous presence of one in the latter and vice versa. Moreover, negative interference between both opposite translocated segments is readily deduced. The simultaneous presence of chiasmata in both distal segments is more frequent than expected if no chiasmata are present in interstitial regions.

Table 4 Grouping of configuration types on the basis of the pairing behaviour in the four end segments only

\begin{tabular}{|c|c|c|c|c|c|}
\hline \multirow{2}{*}{\multicolumn{2}{|c|}{$\begin{array}{l}\text { Configuration by } \\
\text { no. paired end } \\
\text { segments }\end{array}$}} & \multicolumn{2}{|c|}{ 3DL/4DS } & \multicolumn{2}{|c|}{$1 \mathrm{AL} / \mathrm{UN}$} \\
\hline & & \multirow{2}{*}{$\begin{array}{l}\text { Obs. } \\
321\end{array}$} & \multirow{2}{*}{$\begin{array}{l}\text { Exp. } \\
220 \cdot 9\end{array}$} & \multirow{2}{*}{$\frac{\text { Obs. }}{0}$} & \multirow{2}{*}{$\begin{array}{l}\text { Exp. } \\
36 \cdot 4\end{array}$} \\
\hline I & Four & & & & \\
\hline II & Three & 95 & 305 & 143 & 133 \\
\hline III & Two adjacent & 8 & 30.4 & 22 & 101 \\
\hline IV & Two opposite & 246 & $106 \cdot 3$ & 402 & $231 \cdot 2$ \\
\hline \multirow[t]{3}{*}{ V } & One +zero & 2 & $9 \cdot 4$ & 33 & $98 \cdot 4$ \\
\hline & Total & 672 & 672 & 600 & 600 \\
\hline & $\chi^{2}$ & \multicolumn{2}{|c|}{$\begin{array}{l}395.87 \\
P<0.001\end{array}$} & \multicolumn{2}{|c|}{$\begin{array}{l}233.55 \\
P<0.001\end{array}$} \\
\hline
\end{tabular}


Table 5 Grouping of configuration types in which the pairing behaviour in the translocated and interstitial segments is considered

\begin{tabular}{|c|c|c|c|c|c|c|}
\hline \multicolumn{3}{|c|}{ No. paired segments } & \multirow{2}{*}{\multicolumn{2}{|c|}{$3 \mathrm{DL} / 4 \mathrm{DS}$}} & \multirow{2}{*}{\multicolumn{2}{|c|}{$1 \mathrm{AL} / \mathrm{UN}$}} \\
\hline \multirow{2}{*}{$\begin{array}{l}\text { Trans. } \\
\left(\begin{array}{ll}P & N\end{array}\right)\end{array}$} & \multirow{2}{*}{+} & \multirow{2}{*}{$\begin{array}{l}\text { Interst. } \\
\left(\begin{array}{ll}S & T\end{array}\right)\end{array}$} & & & & \\
\hline & & & Obs. & Exp. & Obs. & Exp. \\
\hline 2 & + & 2 & 0 & $11 \cdot 4$ & 0 & $9 \cdot 1$ \\
\hline 2 & + & 1 & 0 & $206 \cdot 9$ & 0 & $21 \cdot 9$ \\
\hline 2 & + & 0 & 337 & $12 \cdot 7$ & 174 & $12 \cdot 6$ \\
\hline 1 & + & 2 & 0 & $16 \cdot 1$ & 0 & $49 \cdot 4$ \\
\hline 1 & + & 1 & 0 & $113 \cdot 7$ & 0 & 119 \\
\hline 1 & + & 0 & 88 & $197 \cdot 6$ & 2 & $68 \cdot 9$ \\
\hline 0 & + & 2 & 124 & $5 \cdot 6$ & 211 & $66 \cdot 4$ \\
\hline 0 & + & 1 & 53 & $38 \cdot 4$ & 159 & 160 \\
\hline 0 & + & 0 & 70 & $67 \cdot 6$ & 54 & $92 \cdot 6$ \\
\hline Total & & & 672 & 672 & 600 & 600 \\
\hline
\end{tabular}

Our results on the existence of both positive and negative chiasma interference between the regions around the translocation break-point are in agreement with similar observations in Acrididae (Henderson, 1963; Fox, 1973; Arana et al., 1980), in Truxaline grasshoppers (Southern, 1967), in mouse, Mus musculus (Leonard and Deknudt, 1967; Leonard, 1971) and in plant material such as in Trillium (Huskins and Newcombe, 1941; Newcombe, 1941) and rye, Secale cereale L. (Sybenga, 1975; Sybenga and Mastenbroek, 1980).

Acknowledgments The authors thank the CAICYT of Spain for the award of a grant (No. 1558-82) to support this work.

\section{REFERENCES}

ARANA, P., SANTOS, J. L. AND GIRALDEX, R. 1980. Chiasma interference and centromere co-orientation in a spontaneous translocation heterozygote of Euchortippus pulvinatus gallicus (Acrididae: Ortopthera). Chromosoma, 78, 327-340.

ARMSTRONG, K. C. 1982. N-banding in Triticum aestivum following Feulgen hydrolisis. Theor. Appl. Genet., 61, 337-339.

BURUCHIN, F. AND LADIZINSKY, G. 1983. Centromere orientation at metaphase I of a translocation heterozygote in lentil. Can. J. Genet. Cytol., 25, 547-553.

ENDRIZZI, J. E. AND KOHEL, R. J. 1966. Use of telosomes in mapping three chromosomes of cotton. Genetics, 54, 535550.

ENDO, T. R. AND GILL, B. S. 1984. Somatic karyotype, heterochromatin distribution and nature of chromosome differentiation in common wheat, Triticum aestivum L. em Thell. Chromosoma, 89, 361-369.
FERRER, E., GONZALEZ, J. M. AND JOUVE, N. $1984 a$. Identification of C-banded chromosomes in meisosis of common wheat, Triticum aestivum L. Theor. Appl. Genet., 67, 257-261.

FERRER, E., GONZALEZ, J. M. AND JOUVE, N. 1984b. The meiotic pairing of nine wheat chromosomes. Theor. Appl. Genet., 69, 193-198.

FoX, D. P. 1973. The control of chiasma distribution in the locust Schistocerca gregaria (Forksal). Chromosoma, 43, 289-328.

GERLACH, W. L. 1977. N-banded karyotypes of wheat species. Chromosoma, 62, 49-56.

GILl, B. S. AND KIMBER, G. 1974. Giemsa C-banding and the evolution of wheat. Proc. Nat. Acad. Sci., 71, 4086-4090.

HENDERSON, S. A. 1963. Chiasma distribution at diplotene in a locust. Heredity, 18, 173-190.

HUSKINS, C. L. AND NEWCOMBE, H. B. 1941. An analysis of chiasma pairs showing chromatid interference in Trillium erectum L. Genetics, 26, 101-127.

IORDANSKY, A. B. ZURABISHVILI, T. G., AND BADAEV, N. S. 1978. Linear differentiation of cereal chromosomes. III. Rye, triticale and 'Aurora' variety. Theor. Appl. Genet., 51, 281-288.

JEWELL, D. C. 1979. Chromosome banding in Triticum aestivum cv. 'Chinese Spring' and Aegilops variabilis. Chromosoma, $71,129-134$.

JOUVE, N., DIEZ, N. AND RODRIGUEZ, M. 1980. C-banding in $6 x$-triticale $\times$ Secale cereale $\mathrm{L}$. Hybrid cytogenetics. Theor. Appl. Genet., 57, 75-79.

JOUVE, N., GONZALEZ, J. M., FOMINAYA, A. AND FERRER, E. 1985. The analysis of meiosis of the B genome in common wheat. Can. J. Genet. Cytol., 27, 17-22.

LEONARD, A. 1971. Radiation-induced translocations in spermatogonia of mice. Mutation Res., 11, 71-88.

LEONARD, A. AND DEKNUDT, G. 1967. Chromosome rearrangements induced in the mouse by embryonic $X$-irradiation. I. Pronuclear stage. Mutation Res., 4, 689-697.

NEWCOMBE, H. B. 1941. Chiasma interference in Trillium erectum. Genetics, 26, 128-136.

SALLEE, P. J. AND KIMBER, G. 1979. An analysis of the pairing of wheat telocentric chromosomes. Proc. 5th Int. Wheat. Genet. Symp. New Delhi, pp 408-419.

SEAL, A. G. 1982. C-banded wheat chromosomes in wheat and triticale. Theor. Appl. Genet., 63, 39-47.

SEARS, E. R. 1954. The aneuploids of common wheat. Mo. Agric. Exp. Stn. Res. Bull., 572, 1-59.

SEARS, E. R. 1972. Chromosome engineering in wheat. Stadler Genet. Symp., 4, 23-28.

SOUTHERN, D. H. 1967. Chiasma distribution in Truxaline grasshoppers. Chromosoma, 22, 164-191.

SYBENGA, J. 1966. The quantitative analysis of chromosome pairing and chiasma formation based on the relative frequencies of MI configuration. IV. Interchange heterozygotes. Genetica, 37, 199-206.

SYBENGA, J. 1970. Simultaneous negative and positive chiasma interference across the break point in interchange heterozygotes. Genetica, 41, 209-230.

SYBENGA, J. 1975. Meiotic Configurations. Springer-Verlag, Berlin.

SYBENGA, J. AND MASTENBROEK, I. 1980. Combined genetic and cytological analysis of positive and negative interference in a interchange heterozygote of rye (Secale cereale L.). Heredity, 44, 83-92.

VAN NIEKERK, H. A. AND PIENAAR, R. v. 1983. Morphology and linear C-band differentiation of Triticum aestivum $\mathrm{L}$ em Thell v. aestivum cv. 'Chinese Spring' chromosomes. Cereal Res. Common., 11, 115-122. 